\title{
Aplicação de modelos lineares de efeitos mistos para avaliar a densidade básica da madeira de duas espécies e um híbrido de Eucalyptus
}

\author{
Linear mixed model applied to evaluate the basic wood density of two \\ species and a hybrid of Eucalyptus
}

\author{
Rogério Antonio de Oliveira ${ }^{1}$ (D), Gislaine Cristina Batistela² (1), Danilo Simões ${ }^{2}$ (D), \\ Carlos Roberto Padovani ${ }^{1}$ (1) \\ ${ }^{1}$ Universidade Estadual Paulista - UNESP, Botucatu, SP, Brasil \\ ${ }^{2}$ Universidade Estadual Paulista - UNESP, Itapeva, SP, Brasil
}

Como citar: Oliveira, R. A., Batistela, G. C., Simões, D., \& Padovani, C. R. (2021). Aplicação de modelos lineares de efeitos mistos para avaliar a densidade básica da madeira de duas espécies e um híbrido de Eucalyptus. Scientia Forestalis, 49(129), e3201. https://doi.org/10.18671/scifor.v49n129.03

\begin{abstract}
Resumo
A qualidade dos produtos derivados da madeira de reflorestamento está ligada diretamente à sua densidade observada nos troncos das árvores. Devido a correlação entre as densidades observadas ao longo dos discos coletados no sentido longitudinal do tronco, modelos lineares de efeitos mistos foram ajustados para avaliar melhor a dependência vertical da densidade básica da madeira em três espécies de eucaliptos: Eucalyptus grandis, Eucalyptus saligna e um híbrido de Eucalyptus grandis x Eucalyptus urophylla. Os resultados dos coeficientes de variação mostraram que a espécie Eucalyptus grandis apresentou a maior variabilidade na densidade básica dos discos ao longo do sentido longitudinal do tronco e as menores médias de densidades em relação às demais espécies; o híbrido possui a maior densidade básica da madeira observada nos discos, embora não apresente muita variabilidade ao longo do tronco. O modelo linear de efeitos mistos mostrou ser uma das ferramentas estatísticas para análise de dados com medidas repetidas, pois a correlação entre as medidas das densidades básicas ao longo do tronco deve ser sempre considerada na análise estatística dos dados. A matriz de correlação não estruturada foi a que melhor se adequou à heterogeneidade da densidade de madeira observada nos discos ao longo do tronco das árvores. Como interpretação do modelo, pode-se concluir que se o intuito é produzir árvores de reflorestamento com maior densidade básica de madeira com menor variabilidade ao longo de seu tronco, o híbrido é a árvore que melhor atende suas necessidades, quando comparada às duas outras espécies.
\end{abstract}

Palavras-chave: Qualidade de madeira; Medidas repetidas; Análise de regressão.

\begin{abstract}
The quality of products derived from reforestation woods is directly linked to the density observed in tree trunks. Due to the natural correlation between the densities collected along the longitudinal trunk discs, linear mixed models were adjusted to better study the vertical dependence of the wood basic density on three eucalyptus: Eucalyptus grandis, Eucaluptus saligna and a hybrid version of Eucalyptus. grandis $\mathrm{x}$ Eucalyptus urophylla. The species Eucalyptus. grandis has shown the greatest variability in the density of the discs along the trunk and also the lowest densities in relation to the other species. The hybrid had the highest density in the discs, although there was not much variability along the trunk. The linear mixed effects model proved to be one of the statistical tools for data analysis with repeated measurements, since the correlation between measurements of basic densities along the trunk should always be considered in the statistical analysis of the data. The unstructured correlation matrix was the best suited
\end{abstract}

Fonte de financiamento: Nenhuma.

Conflito de interesse: Nada a declarar.

Autor correspondente: rogerio.oliveira@unesp.br

Recebido: 30 novembro 2018.

Aceito: 3 abril 2020.

Editor: Paulo Henrique Müller Silva.

(c) Este é um artigo publicado em acesso aberto (Open Access) sob a licença Creative Commons Attribution, que permite uso, distribuição e reprodução m qualquer meio, sem restrições desde que o trabalho original seja corretamente citado. 
to the heterogeneity of wood density observed in the discs along the tree trunk. The interpretation of the model showed that if the intention is to produce reforestation trees with higher basic wood density and lower variability along its trunk, the hybrid is the best choice when compared to the other two species.

Keywords: Wood quality; Repeated measures; Analysis of regression.

\section{INTRODUÇÃO}

A qualidade dos produtos madeireiros pode ser influenciada principalmente pela densidade básica, consequentente, torna-se uma premissa para os processos produtivos das empresas de base florestal. Contudo, a densidade básica da madeira é influenciada por diversos fatores, como exemplo, idade, espécies florestais, volume médio individual do tronco, tratos silviculturais, local de retirada dos discos no tronco no sentido longitudinal, dentre outros.

Desse modo, existe a possibilidade de a densidade básica apresentar variabilidade entre espécies de árvores de um mesmo gênero, entre indivíduos de uma mesma espécie ou ainda dentro do tronco de uma mesma árvore. Nesta perspectiva, a análise da densidade básica da madeira mensurada ao longo do sentido longitudinal do tronco da árvore caracteriza-se como uma estrutura de medidas repetidas, pois comumente apresenta uma dependência natural de autocorrelação, não atendendo, portanto, a suposição de independência entre as observações, pressuposto necessário para a aplicação dos modelos clássicos de análise de variância e regressão.

Isto posto, uma das técnicas que pode ser aplicada para avaliar a densidade de forma plausível é a construção de modelos lineares de efeitos mistos. Toda a teoria e aplicação dos modelos lineares de efeitos mistos foram discutidos por diferentes pesquisadores, incluindo Diggle (1988), Milliken \& Johnson (1992), Diggle et al. (1994), Davidian \& Giltinan (1995), Verbeke \& Molenberghs (1997, 2000), Vonesh \& Chinchilli (1997), Demidenko (2004), Molenberghs \& Verbeke (2005) e Littell et al. (2006).

Ao longo das últimas três décadas, os modelos lineares clássicos foram adaptados para incluir também os efeitos aleatórios, além dos tradicionais efeitos fixos, criando assim a classe dos modelos lineares de efeitos mistos. Estes modelos são bastante úteis quando há medidas repetidas na mesma unidade amostral (dados longitudinais) ou quando as medidas são realizadas dentro de um mesmo conjunto de unidades amostrais homogêneas, agrupadas por algum método de agrupamento. Portanto, os modelos lineares mistos permitem certa flexibilidade na modelagem não apenas nas médias, mas também nas estruturas de variâncias e covariâncias do vetor de erros do modelo. Ademais, o nome de modelos de efeitos mistos é devido ao fato do modelo linear conter parâmetros de efeitos fixos e aleatórios (Henderson, 1990; Searle et al., 1992).

Hoeting et al. (2006) apresentaram uma revisão e discussão dos aspectos teóricos relacionados aos critérios de seleção dos modelos ajustados, destacando os critérios Akaike Information Criterion (AIC) e Akaike's Information Corrected Criterion $\left(A I C_{C}\right)$ e indicam que o melhor modelo será aquele que apresentar menor valor de $\mathrm{AIC}$ e $\mathrm{AlC}_{\mathrm{C}}$, pois apresenta $\mathrm{O}$ melhor ajuste doconjunto de dados dentre todos os modelos avaliados.

Após escolher o melhor modelo linear de efeitos mistos, uma análise de diagnóstico de influência das observações precisa ser realizada para verificar os comportamentos dos resíduos gerados pelo ajuste do modelo. A ideia geral é quantificar a influência de uma ou mais observações nos cálculos das estimativas dos parâmetros do modelo, baseando-se no conjunto global de dados, em que, remove-se uma observação do conjunto de dados e reajusta-se novamente o modelo com as observações restantes. Fundamentalmente, observam-se as diferenças encontradas entre as estimativas dos parâmetros obtidas no modelo ajustado com os dados sem a observação removida.

As estatísticas de influência podem ser divididas pelo objetivo de diagnóstico, ou seja: mensurar as diferenças encontradas entre a função de verossimilhança do modelo com todas as árvores e a função de verossimilhança com a retirada de cada uma das observações, avaliando o impacto individual de cada árvore na função de verossimilhança do modelo (Cook 
\& Weisberg, 1982); mensurar as influências nas estimativas dos parâmetros utilizando a Distância de Cook (Cook, 1977, 1979); verificar a influência na precisão das estimativas, qual seja representada pela razão entre os determinantes das matrizes de covariância dos parâmetros ajustados sem a observação e da covariância (CovRatio) dos parâmetros com todos os dados (SAS Institute Inc., 2012).

Considerando algumas aplicações dos modelos lineares de efeitos mistos em dados relacionados às ciências florestais, pode-se encontrar nos trabalhos apresentados por Santos et al. (2004) e Calegario et al. (2005) modelos lineares mistos para avaliar a qualidade de madeira serradas nas progênies de Eucalyptus grandis, inclusive com a taxa de crescimento dos clones desta variedade de eucaliptos. Guilley et al. (2004) apresentaram também uma análise de modelos lineares mistos para determinar a densidade média da madeira de carvalhos (Quercus petraea Liebl). Repola (2006) apresentou análises de modelos mistos para determinar a densidade de madeira em Pinus sylvestris, Picea abies, Betula pendula e Betula pubescens.

À vista disso, objetivou-se ajustar modelos lineares de efeitos mistos para avaliar a densidade básica da madeira de Eucalyptus determinadas ao longo do tronco das árvores, na direção longitudinal, supondo diferentes estruturas de correlação para o ajuste de modelos mistos, para duas espécies e um clone híbrido de Eucalyptus.

\section{MATERIAL E MÉTODOS}

\section{Suporte do estudo}

Foram utilizados dados relativos a 150 árvores de florestas de Eucalyptus, com oito anos de idade, plantanda em três talhões diferentes que possuíam 250 árvores, sendo de cada talhão selecionadas 50 árvores de Eucalyptus grandis, 50 de Eucalyptus saligna e 50 de um clone híbrido de Eucalyptus grandis x Eucalyptus urophylla, com alturas médias 30,42 m, 23,51 m e 29,72 m, respectivamente.

Deste modo, foi constituído o experimento clonal, localizado nas coordenadas geográficas $23^{\circ} 58^{\prime}$ de Latitude Sul e $48^{\circ} 03^{\prime}$ de Longitude Oeste, no Estado de São Paulo, Brasil. As características climáticas da região são classificadas como Cwa clima tropical de altitude, com chuvas no verão e seca no inverno, com a temperatura média do mês mais quente superior a $22^{\circ} \mathrm{C}$ (Alvares et al., 2013).

A densidade básica da madeira foi determinada para cada indivíduo a partir de discos localizados nas posições a 0\% (base), diâmetro à altura do peito (DAP), 25\%,50\%,75\%, 100\% da altura comercial (diâmetro igual a $5 \mathrm{~cm}$ ), respectivamente, discos A, B, C, D, E e F, que, por conseguinte, a altura de corte dos discos variou para cada tronco, baseada no comprimento de cada indivíduo arbóreo. Ademais, para as seis posições foram coletadas amostras para a determinação da densidade básica da madeira $\left(\mathrm{g} / \mathrm{cm}^{3}\right) \cdot e m$ consonância à metodologia utilizada por Tomazello Filho (1985) e Benjamin \& Ballarin (2003).

\section{Desenho do experimento}

O modelo para explicar a variação da resposta média da densidade básica da madeira de Eucalyptus foi ajustado considerando o vetor de dados composto pelas mensurações realizadas em discos das seis posições ( $\mathrm{A}$ a F) de uma mesma árvore (unidade experimental), caracterizando medidas repetidas na mesma unidade (dados longitudinais).

O modelo linear de efeitos mistos utilizado nesse trabalho foi expresso como

$$
y=X \beta+Z \gamma+\varepsilon,
$$

em que y é o vetor da variável resposta (densidade básica da madeira), $\mathbf{X}$ e $\boldsymbol{\beta}$ são, respectivamente, a matriz conhecida (composta por variáveis categóricas ou contínuas) e o vetor de parâmetros desconhecido de efeitos fixos, semelhante ao modelo linear 
generalizado e $\boldsymbol{\varepsilon}$ é o vetor de erros. Entretanto, este modelo possui a adição de uma matriz conhecida $\mathbf{Z}$ e de um vetor desconhecido de efeitos aleatórios denotado por $\boldsymbol{y}$. A matriz $\mathbf{Z}$ pode conter variáveis contínuas ou categorizadas, dependo do conjunto de dados utilizado para a análise estatística.

Para compreender a relação entre a modelagem da variância nos modelos mistos, assume-se que $\boldsymbol{y}$ e $\boldsymbol{\varepsilon}$ são variáveis aleatórias normais não correlacionadas com médias nulas e variâncias iguais a $\mathbf{G}$ e $\mathbf{R}$, respectivamente. Logo, a estimação é um pouco mais complexa nos modelos mistos que nos modelos lineares generalizados, pois além de estimar o vetor $\boldsymbol{\beta}$, é necessário estimar também os outros parâmetros desconhecidos de $\mathbf{y}, \mathbf{G}$ e R. A estimação é realizada pelo método de mínimos quadrados generalizados, conforme solução apresentada por Henderson (1984) .

A Tabela 1 apresenta as principais estruturas de correlação $\mathbf{R}$, consideradas nos ajustes de modelos mistos. Antes de ajustar o modelo, estima-se os parâmetros da matriz de correlação das medidas de densidade básica coletadas ao longo do tronco.

Tabela 1. Estruturas de matrizes $\mathbf{R}$ para análise de modelos mistos.

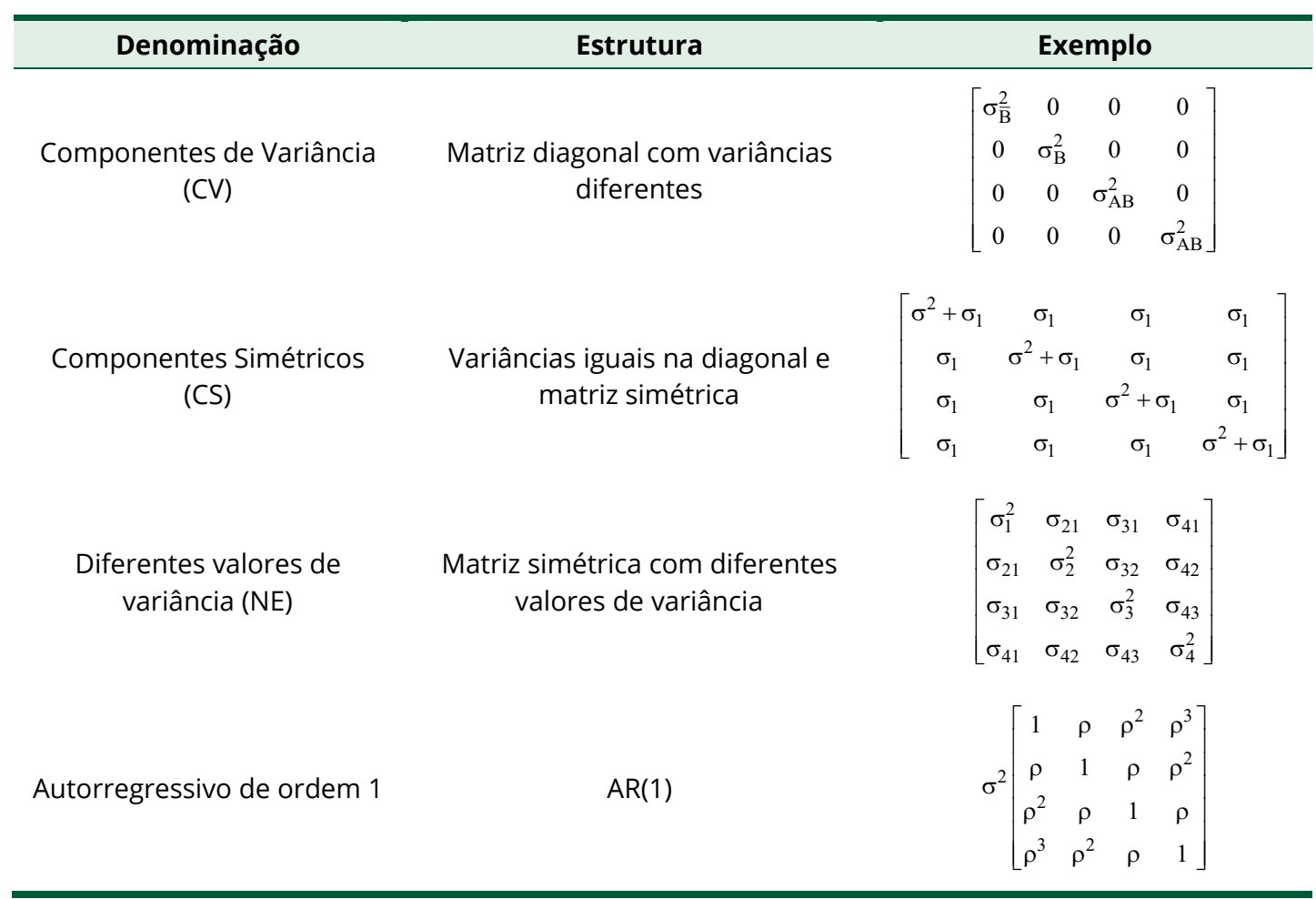

Os ajustes dos modelos lineares com modelos de efeitos mistos e as análises das influências das observações nas estimativas dos parâmetros do modelo final foram realizados por meio do procedimento MIXED do software SAS, versão 9.3 (SAS Institute Inc., 2012).

\section{RESULTADOS E DISCUSSÃO}

Os valores da densidade básica da madeira do híbrido Eucalyptus grandis $x$ Eucalyptus urophylla apresentaram maiores medianas em relação as outras duas espécies, conforme os resultados da Tabela 2.

Pela Figura 1, notou-se que a espécie Eucalyptus saligna apresentou cinco valores discrepantes superiores em relação aos demais, para as medidas observadas nos discos B, C e $F$, enquanto que verificou-se a maior variabilidade nos valores de densidade básica da espécie Eucalyptus grandis. 
Tabela 2.Estatistica Descritiva da densidade básica $\left(\mathrm{g} / \mathrm{cm}^{3}\right)$ para as duas espécies e o híbrido de eucaliptos.

\begin{tabular}{|c|c|c|c|c|c|c|c|}
\hline Espécie & Disco & $\begin{array}{l}\text { Média } \\
\left(\mathrm{g} / \mathrm{cm}^{3}\right)\end{array}$ & $\begin{array}{l}\text { Desvio } \\
\text { Padrão }\end{array}$ & $\begin{array}{c}\text { Coeficiente } \\
\text { Variação (\%) }\end{array}$ & $\begin{array}{l}\text { Mínimo } \\
\left(\mathrm{g} / \mathrm{cm}^{3}\right)\end{array}$ & $\begin{array}{l}\text { Mediana } \\
\left(\mathrm{g} / \mathrm{cm}^{3}\right)\end{array}$ & $\begin{array}{c}\text { Máximo } \\
\left(\mathrm{g} / \mathrm{cm}^{3}\right)\end{array}$ \\
\hline \multirow{6}{*}{ Eucalyptus grandis } & $0 \%$ & 0,461 & 0,044 & 9,600 & 0,386 & 0,464 & 0,567 \\
\hline & DAP & 0,450 & 0,039 & 8,690 & 0,370 & 0,441 & 0,511 \\
\hline & $25 \%$ & 0,461 & 0,044 & 9,590 & 0,370 & 0,452 & 0,556 \\
\hline & $50 \%$ & 0,456 & 0,047 & 10,300 & 0,386 & 0,454 & 0,547 \\
\hline & $75 \%$ & 0,454 & 0,044 & 9,770 & 0,368 & 0,467 & 0,539 \\
\hline & $100 \%$ & 0,459 & 0,051 & 11,160 & 0,367 & 0,458 & 0,569 \\
\hline \multirow{6}{*}{$\begin{array}{c}\text { Híbrido Eucalyptus } \\
\text { grandis } x \text { Eucalyptus } \\
\text { urophylla }\end{array}$} & $0 \%$ & 0,493 & 0,030 & 6,040 & 0,442 & 0,491 & 0,553 \\
\hline & DAP & 0,489 & 0,039 & 8,020 & 0,430 & 0,485 & 0,576 \\
\hline & $25 \%$ & 0,500 & 0,033 & 6,670 & 0,440 & 0,495 & 0,566 \\
\hline & $50 \%$ & 0,494 & 0,030 & 6,080 & 0,434 & 0,494 & 0,547 \\
\hline & $75 \%$ & 0,490 & 0,041 & 8,300 & 0,420 & 0,493 & 0,573 \\
\hline & $100 \%$ & 0,502 & 0,040 & 7,960 & 0,417 & 0,502 & 0,596 \\
\hline \multirow{6}{*}{ Eucalyptus saligna } & $0 \%$ & 0,486 & 0,024 & 4,990 & 0,439 & 0,481 & 0,544 \\
\hline & DAP & 0,476 & 0,025 & 5,190 & 0,432 & 0,477 & 0,556 \\
\hline & $25 \%$ & 0,470 & 0,025 & 5,240 & 0,430 & 0,469 & 0,563 \\
\hline & $50 \%$ & 0,481 & 0,027 & 5,550 & 0,434 & 0,476 & 0,538 \\
\hline & $75 \%$ & 0,465 & 0,024 & 5,180 & 0,406 & 0,462 & 0,527 \\
\hline & $100 \%$ & 0,466 & 0,020 & 4,300 & 0,428 & 0,462 & 0,518 \\
\hline
\end{tabular}

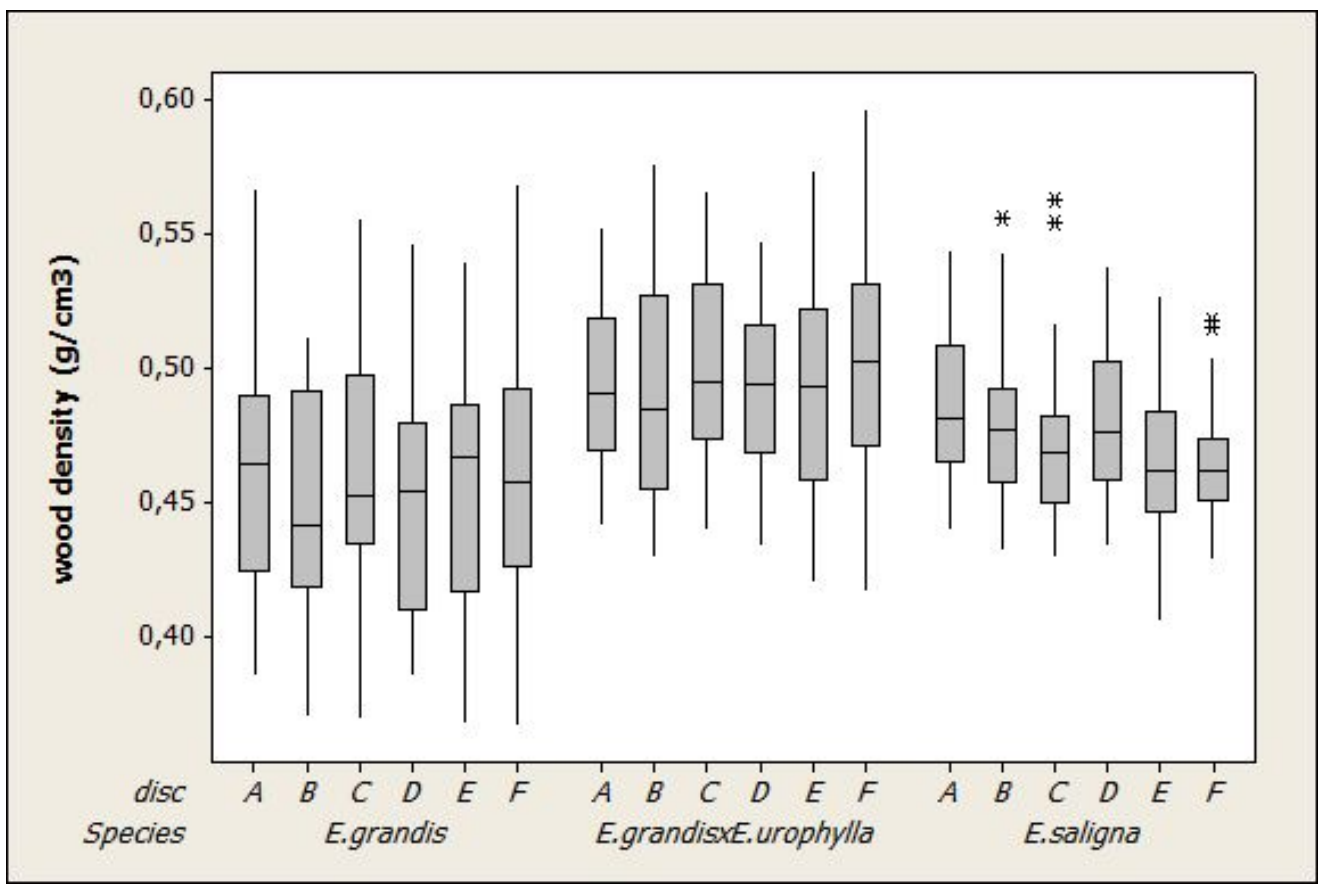

Figura 1. Distribuição da densidade da madeira nos discos as duas espécies e o híbrido de eucaliptos.

Para analisar as densidades básicas observadas nas duas espécies e no híbrido de Eucayptus, foram considerados como efeitos fixos a espécie/híbrido florestal (categorias: Eucalyptus grandis, Eucalyptus saligna e híbrido Eucalyptus grandis $x$ Eucalyptus urophylla) e a altura do disco (categorias: A, B, C, D, E e F); e as árvores foram consideradas como efeito aleatório dentro de cada espécie. 
No modelo foram utilizadas a espécie Eucalyptus saligna e o disco F como referência de suas respectivas categorias, para a construção da matriz de planejamento e estimação dos parâmetros do modelo linear (Missio \& Jacobe, 2007).

Os ajustes dos modelos foram realizados para as seguintes estruturas de matrizes de correlação: não estruturada (NE), autoregressivo de ordem 1 (AR(1)), diagonal da matriz com diferentes variâncias (CV) e componentes simétricos com variâncias iguais (CS). Na escolha do melhor modelo, empregando as diferentes estruturas de matrizes, foi utilizadoo $\mathrm{AIC}$ e $\mathrm{AlC}_{\mathrm{c}}$.

A Tabela 3 apresenta os valores de $\mathrm{AIC}$ e $\mathrm{AlC}_{\mathrm{C}}$ para os diferentes modelos mistos ajustados aos dados da densidade básica da madeira. Por meio do $\mathrm{AIC}$ e $\mathrm{AlC}_{\mathrm{C}}$ o melhor valor de ajuste aos dados foi observado com a matriz não estruturada (NE) para a matriz de covariância, em que a matriz $\mathbf{R}$ apresenta todas as variâncias diferentes, demonstrando uma grande heterogeneidade das medidas repetidas ao longo do tronco das árvores.

Tabela 3. Valores do AIC e do $\mathrm{AIC}_{\mathrm{c}}$ para estruturas de matrizes $\mathbf{R}$.

\begin{tabular}{ccccc}
\hline Medida de ajuste & NE & AR(1) & CS & CV \\
\hline AIC & $-\mathbf{4 0 2 2 . 1}$ & -3898.8 & -3768.5 & -3417.2 \\
AIC $_{C}$ & -4018.5 & -3897.9 & -3767.6 & -3416.3 \\
\hline
\end{tabular}

Os parâmetros estimados do modelo linear misto com a estrutura de matriz não estruturada podem ser observados na Tabela 4. O modelo misto considera a espécie Eucalyptus saligna e o último disco $\mathrm{F}$ como referências de suas categorias de variáveis qualitativas. Observou-se que a maioria das interações entre as duas espécies e o híbrido e disco são estatisticamente significativas $(p<0,05)$, indicando que existem diferenças encontradas nos discos em função destas espécies e do híbrido de eucaliptos.

Tabela 4. Estimativas dos parâmetros do modelo linear misto para a matriz de covariância não estruturada.

\begin{tabular}{|c|c|c|c|c|c|c|}
\hline Efeito fixo & Espécie & disco & Estimativa & Erro padrão & $\mathbf{T}$ & Valor $p$ \\
\hline Intercepto & & & 0,467 & 0,006 & 82,190 & $<0,0001$ \\
\hline Espécie & Eucalyptusgrandis & & $-0,009$ & 0,008 & $-1,100$ & 0,2746 \\
\hline Espécie & Hibrido Eucalyptusgrandis x Eucalyptusurophylla & & 0,037 & 0,008 & 4,630 & $<0,0001$ \\
\hline Espécie & Eucalyptus saligna & & & & & \\
\hline disco & & $0 \%$ & 0,018 & 0,005 & 3,810 & 0,0002 \\
\hline disco & & DAP & 0,008 & 0,006 & 1,340 & 0,1822 \\
\hline disco & & $25 \%$ & 0,000 & 0,007 & 0,000 & 0,9994 \\
\hline disco & & $50 \%$ & 0,014 & 0,005 & 3,040 & 0,0028 \\
\hline disco & & $75 \%$ & $-0,002$ & 0,004 & $-0,440$ & 0,6613 \\
\hline disco & & $100 \%$ & & & & \\
\hline Espécie*disco & Eucalyptusgrandis & $0 \%$ & $-0,022$ & 0,007 & $-3,250$ & 0,0015 \\
\hline Espécie*disco & Eucalyptusgrandis & DAP & $-0,019$ & 0,009 & $-2,090$ & 0,038 \\
\hline Espécie*disco & Eucalyptusgrandis & $25 \%$ & $-0,002$ & 0,010 & $-0,260$ & 0,7959 \\
\hline Espécie*disco & Eucalyptusgrandis & $50 \%$ & $-0,017$ & 0,007 & $-2,600$ & 0,0102 \\
\hline Espécie*disco & Eucalyptusgrandis & $75 \%$ & $-0,004$ & 0,005 & $-0,780$ & 0,4343 \\
\hline Espécie*disco & Eucalyptusgrandis & $100 \%$ & & & & \\
\hline Espécie*disco & Hibrido Eucalyptusgrandis x Eucalyptusurophylla & $0 \%$ & $-0,031$ & 0,007 & $-4,460$ & $<0,0001$ \\
\hline Espécie*disco & Hibrido Eucalyptusgrandis x Eucalyptusurophylla & DAP & $-0,026$ & 0,009 & $-2,960$ & 0,0036 \\
\hline Espécie*disco & Hibrido Eucalyptusgrandis x Eucalyptusurophylla & $25 \%$ & $-0,009$ & 0,010 & $-0,990$ & 0,3248 \\
\hline Espécie*disco & Hibrido Eucalyptusgrandis x Eucalyptusurophylla & $50 \%$ & $-0,024$ & 0,007 & $-3,690$ & 0,0003 \\
\hline Espécie*disco & Hibrido Eucalyptusgrandis x Eucalyptusurophylla & $\mathrm{E}$ & $-0,011$ & 0,005 & $-2,240$ & 0,0267 \\
\hline
\end{tabular}


A Figura 2 mostra que a observação referente ao indivíduo arbóreo 126 apresentou uma grande diferença entre o valor da verossimilhança do modelo estatístico com todas as observações e o modelo sem esta observação. O indivíduo 126 corresponde a um tronco da espécie Eucalyptus grandis, entretanto, não foi notada nenhuma observação discrepante no Box-Plot para esta espécie (Figura 1). Na análise de diagnóstico do modelo, caso este indivíduo influencie também nas estimativas encontradas para os parâmetros do modelo, é importante decidir criteriosamente sua possível exclusão, devido ao fato de ser uma possível observação discrepante e influente.

\section{Likelihood Distance}

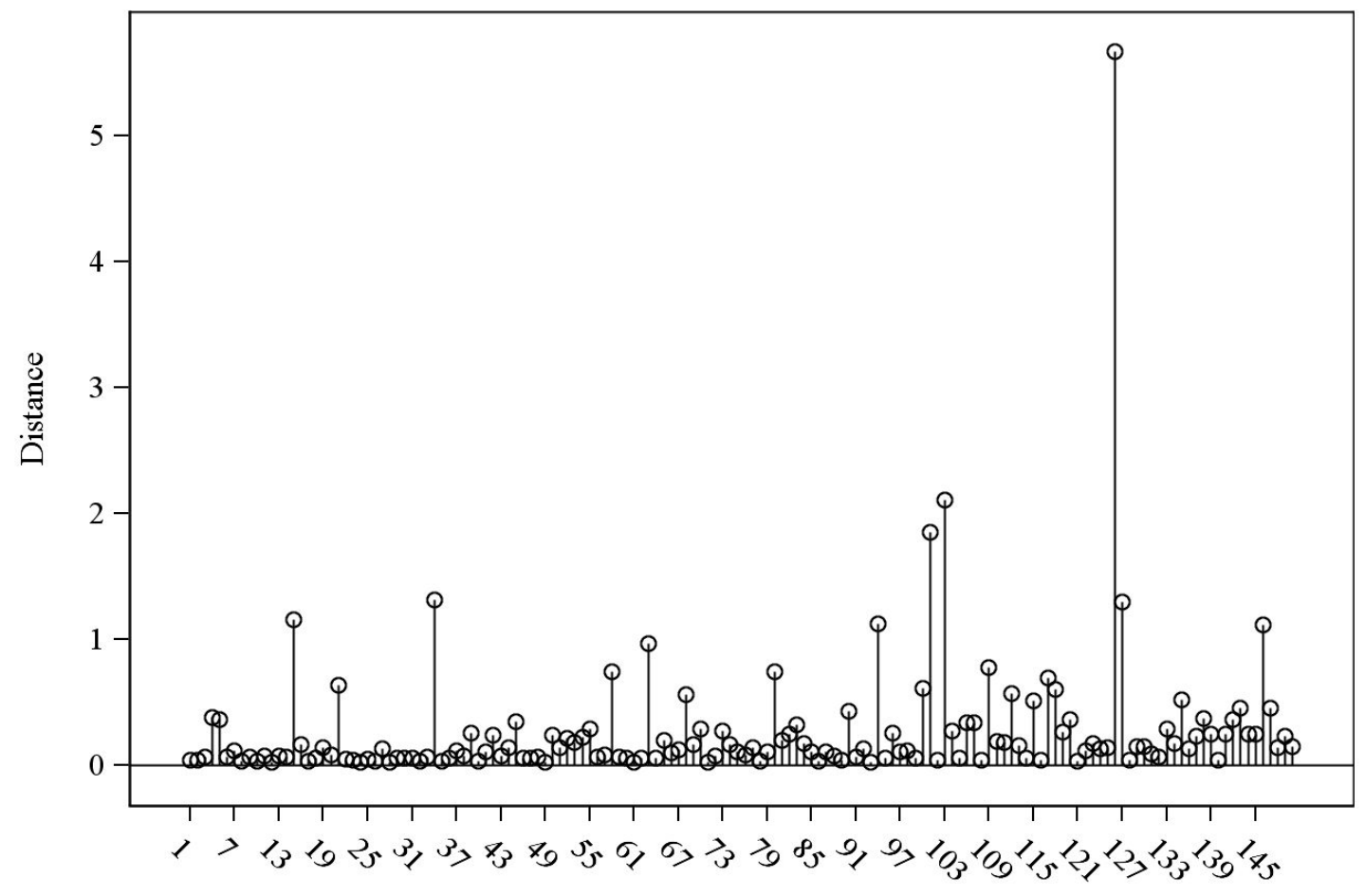

Deleted arvore

Figura 2. Valores individuais para distância de Verossimilhança do melhor modelo misto ajustado.

Pode-se visualizar na Figura 3 que a observação referente ao indivíduo 34 apresentou valor alto para a distância de Cook e para CovRatio em relação as demais observações da densidade básica da madeira das espécies estudadas.

Embora constatando-se que o indivíduo 34 influencia nas estimativas dos parâmetros do modelo (Figuras 4 e 5), fez-se a opção pelo ajuste do modelo com esta observação, porque ela incorpora a alta variabilidade encontrada nas duas espécies e um híbrido de eucaliptos.

A Figura 6 exibe os intervalos de 95\% de confiança para a densidade básica média em cada um dos discos para as duas espécies e o híbrido de eucaliptos. Pode-se ver que existe grande variabilidade nas densidades básicas ao longo do tronco, mas que depende também das espécies e do híbrido considerados. Este fato corroborou o resultado das interações significativas apontadas no modelo entre as espécies e o híbrido e o disco considerado. A espécie Eucalyptus grandis apresentou os menores valores médios de densidade básica da madeira nos discos em comparação a outra espécie e ao híbrido. Entretanto, pode-se ver que o híbrido apresentou as maiores densidades médias de madeira e as menores variabilidades ao longo do tronco. 
Cook's D Fixed Effects

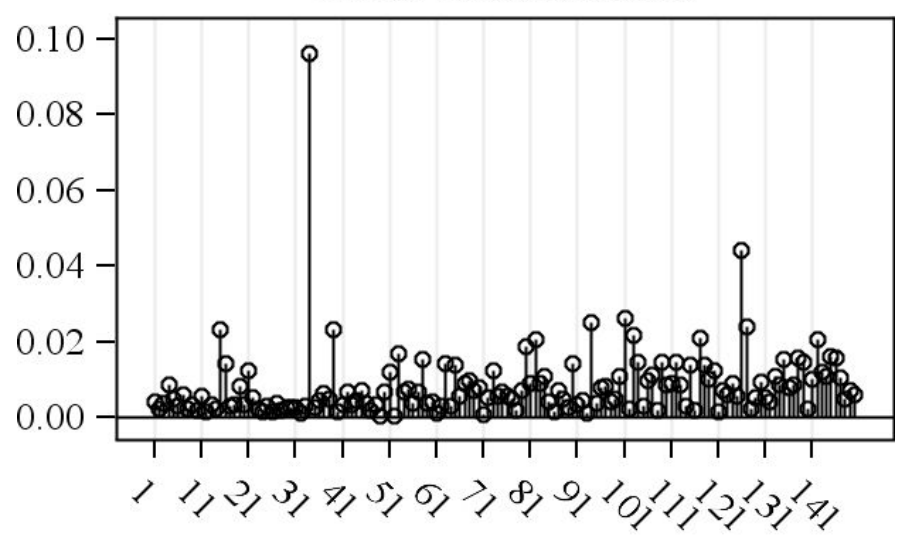

CovRatio Fixed Effects

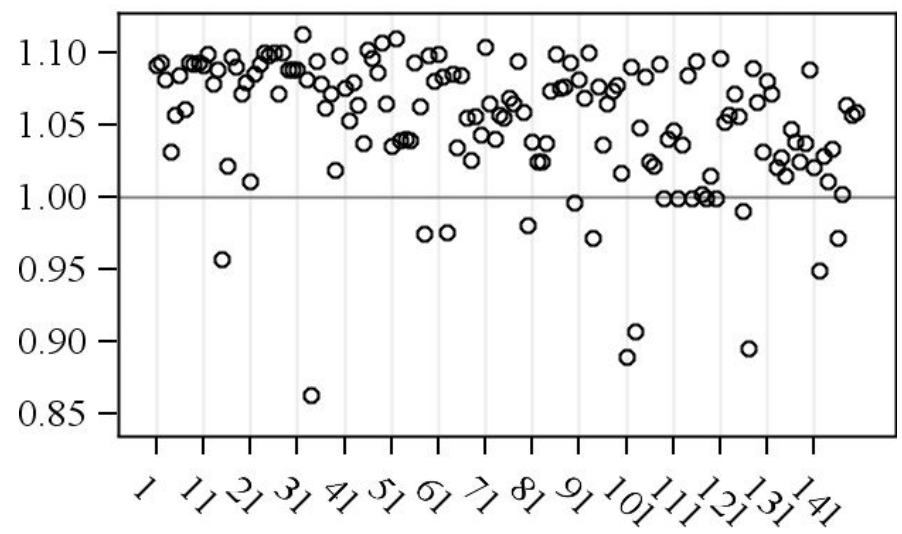

Figura 3. Valores individuais para a distância de Cook e para CovRatio do melhor modelo ajustado.

\section{Fixed Effects Deletion Estimates for densidade}
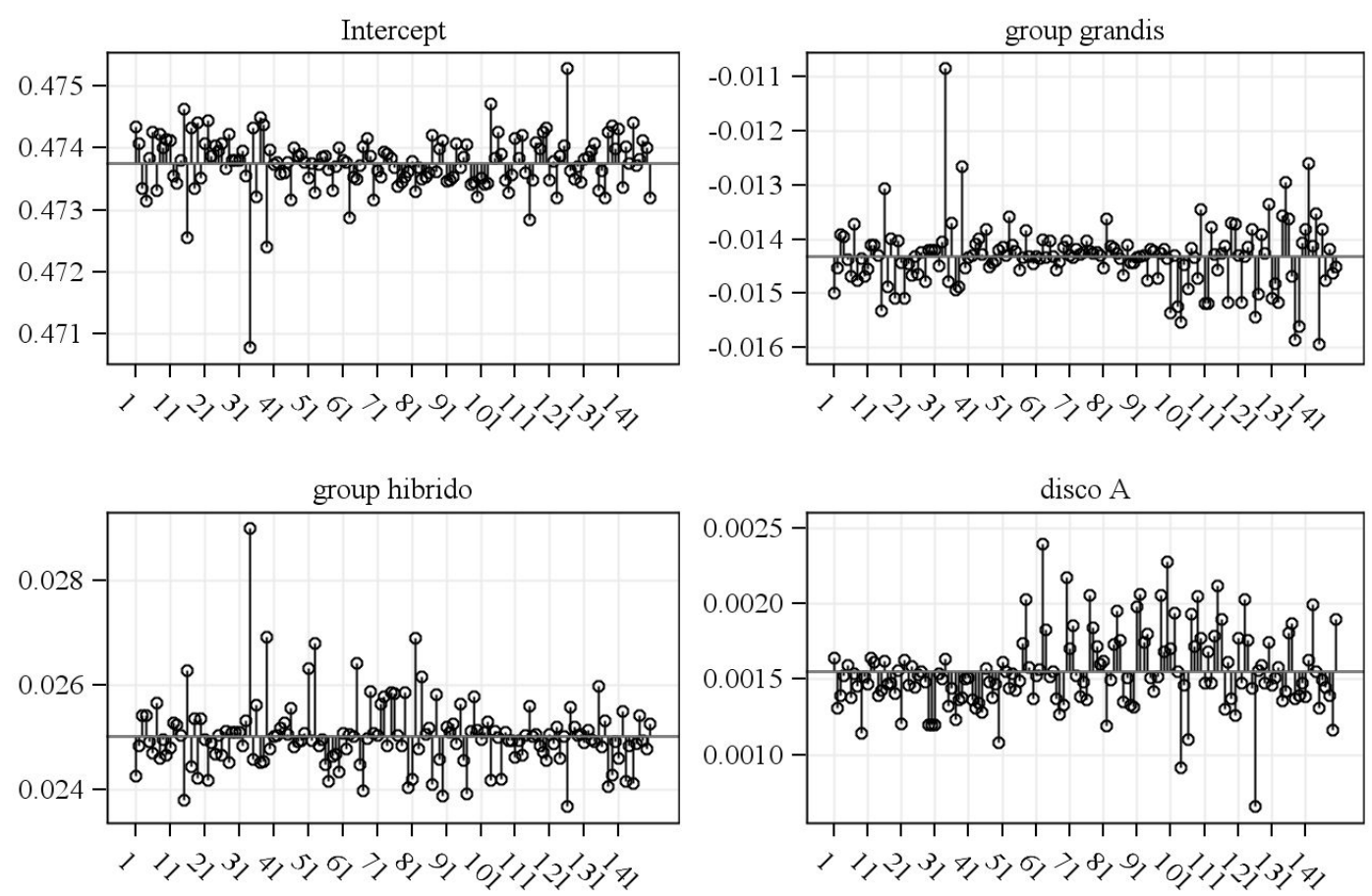

Deleted tree

Figura 4. Valores individuais para a influência nos parâmetros do melhor modelo. 


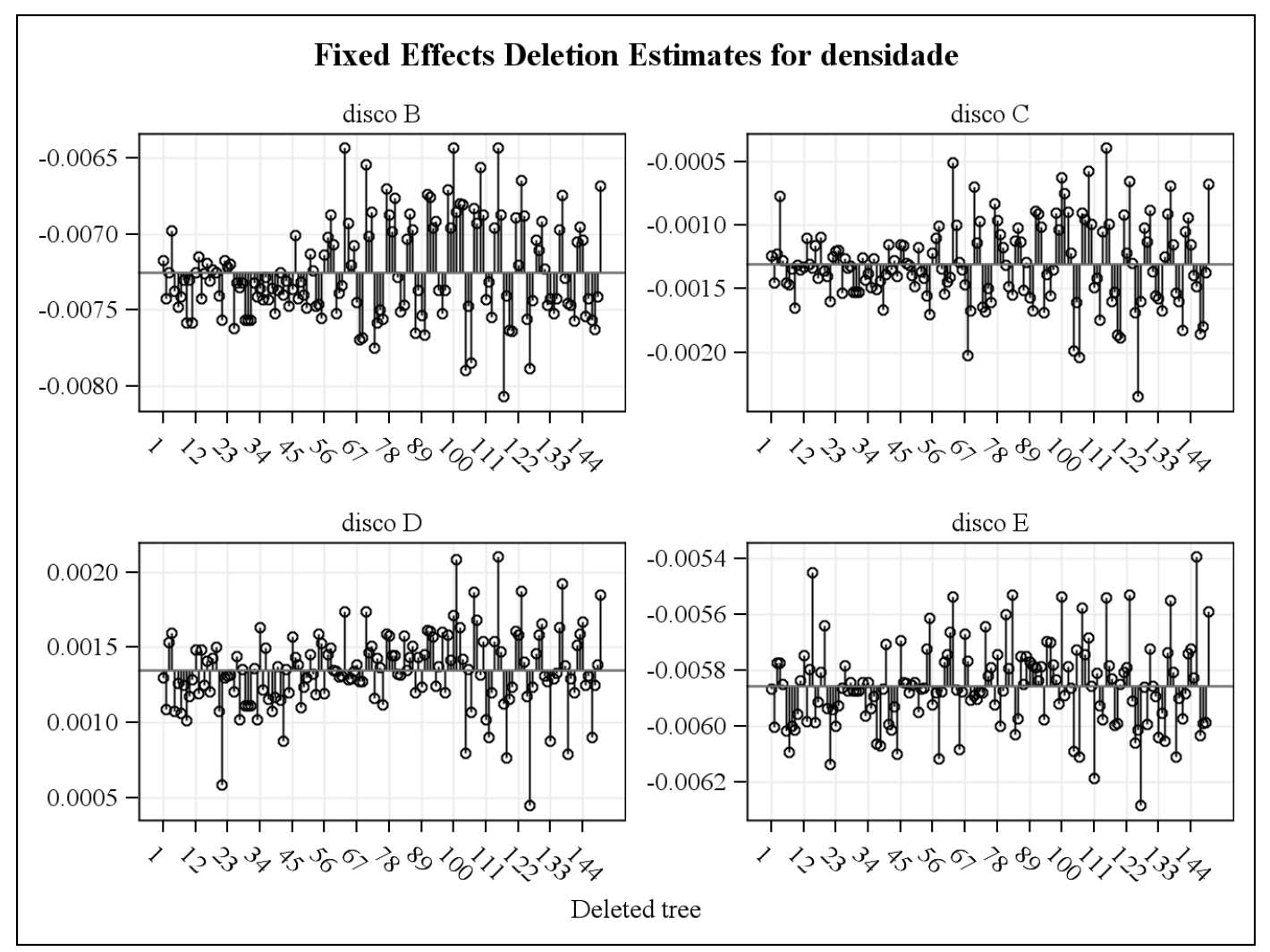

Figura 5. Valores individuais para a influência nos parâmetros do melhor modelo misto.

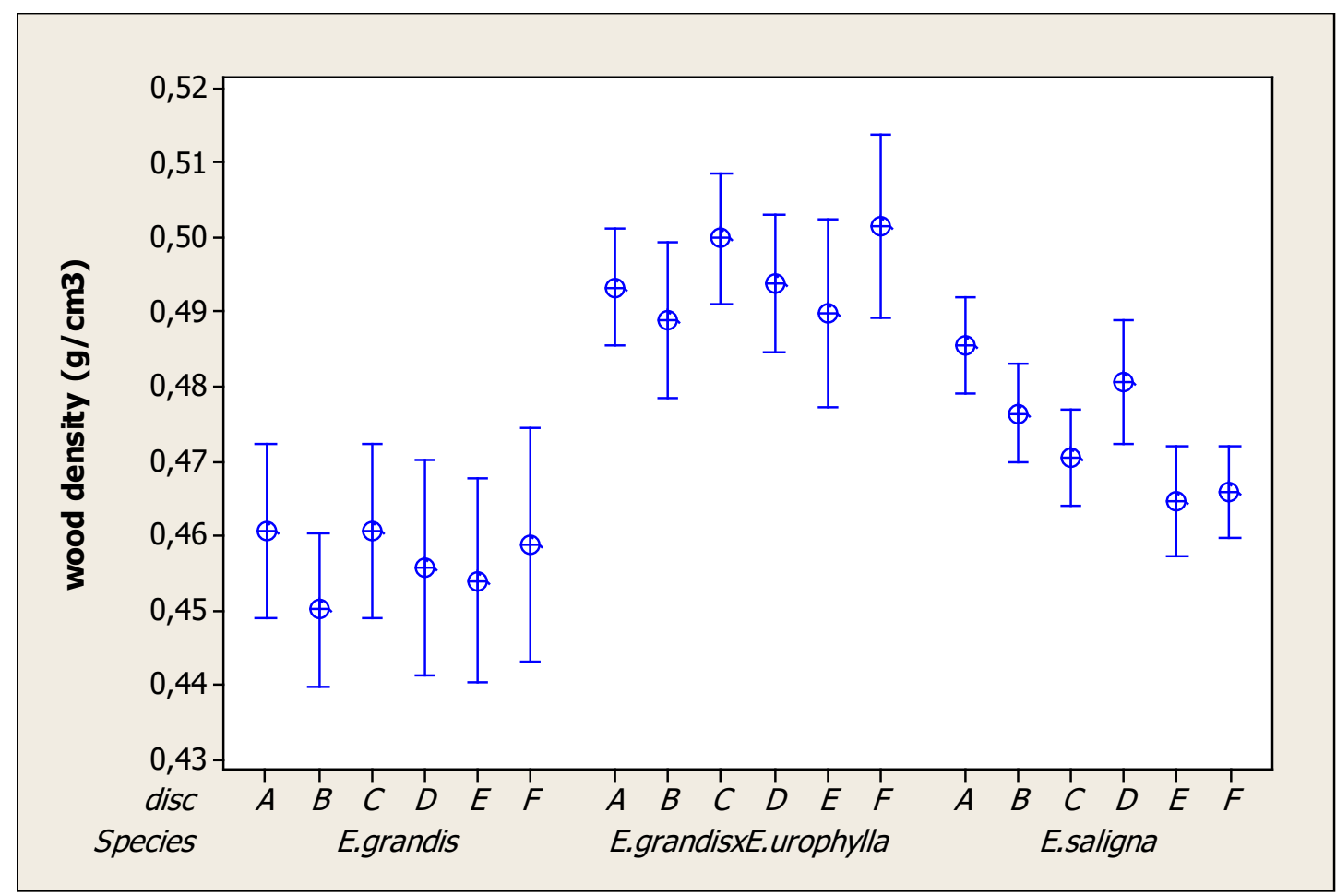

Figura 6. Intervalos de $95 \%$ de Confiança para a densidade básica média $\left(\mathrm{g} / \mathrm{cm}^{3}\right)$ a madeira nos discos das duas espécies e o híbrido de eucaliptos.

\section{CONCLUSÕES}

O modelo linear de efeitos mistos é uma das ferramentas estatísticas para análise de dados com medidas repetidas, pois a correlação entre as medidas das densidades básicas ao 
longo do tronco será sempre considerada na análise estatística. A matriz de correlação não estruturada foi a que melhor se adequou à heterogeneidade da densidade de madeira observada nos discos ao longo do tronco das árvores.

Em resumo, com a demanda crescente por madeira de reflorestamento, o gestor florestal precisa tomar algumas decisões a fim de garantir qualidade da madeira produzida. Se o intuito for produzir madeira com maior densidade básica e com menor variabilidade ao longo do tronco, os híbridos podem fornecer os melhores resultados em relação as espécies Eucalyptus grandis e Eucalyptus saligna.

\section{REFERÊNCIAS}

Alvares, C. A., Stape, J. L., Sentelhas, P. C., Gonçalves, J. L. M., \& Sparovek, G. (2013). Koppen's climate classification map for Brazil. Meteorologishe Zeitschrift, 22(6), 711-728. http://dx.doi.org/10.1127/0941-2948/2013/0507.

Benjamin, C. A., \& Ballarin, A. W. (2003). Correlações entre a densidade básica média ponderada da madeira e a densidade básica em alguns pontos ao longo do tronco de árvores de $E$. saligna, $E$. grandis e híbridos de E. grandis $\times$ E. urophylla. Energia na Agricultura, 18(3), 1-12.

Calegario, N., Daniels, R. F., Souza, A. L., \& Maestri, R. (2005). Estimativa do crescimento de povoamentos de Eucalyptus baseada em modelos lineares em multiníveis de efeitos mistos. Revista Árvore, 29(2), 251-264. http://dx.doi.org/10.1590/S0100-67622005000200008.

Cook, R. D. (1977). Detection of influential observations in linear regression. Technometrics, 19, 15-18.

Cook, R. D. (1979). Influential observations in linear regression. Journal of the American Statistical Association, 74(365), 169-174. http://dx.doi.org/10.1080/01621459.1979.10481634.

Cook, R. D., \& Weisberg, S. (1982). Residuals and influence in regression (240 p.). New York: Chapman \& Hall.

Davidian, M., \& Giltinan, D. M. (1995). Nonlinear models for repeated measurement data (360 p.). New York: Chapman \& Hall.

Demidenko, E. (2004). Mixed models: theory and applications (740 p.). New York: John Wiley. http://dx.doi.org/10.1002/0471728438.

Diggle, P. J. (1988). An approach to the analysis of repeated measurements. Biometrics, 44(4), 959-971. PMid:3233259. http://dx.doi.org/10.2307/2531727.

Diggle, P. J., Liang, K. Y., \& Zeger, S. L. (1994). Analysis of longitudinal data (400 p.). Oxford: Clarendon Press.

Guilley, E., Herve, J. C., \& Nepveu, G. (2004). The influence of site quality, silviculture and region on wood density mixed model in Quercus petraea Liebl. Forest Ecology and Management, 189(1-3), 111-121. http://dx.doi.org/10.1016/j.foreco.2003.07.033.

Henderson, C. (1990). Statistical method in animal improvement: historical overview. In D. Gianola \& K. Hammond (Eds.), Advances in statistical methods for genetic improvement of livestock (Advanced Series in Agriculture Sciences, No. 18, pp. 1-14). New York: Springer-Verlag. http://dx.doi.org/10.1007/9783-642-74487-7_1.

Henderson, C. R. (1984). Applications of linear models in animal breeding (384 p.). Guelph: University of Guelph.

Hoeting, J. A., Davis, R. A., Merton, A. A., \& Thompson, S. E. (2006). Model selection for geostatistical models. Ecological Applications, 16(1), 87-98. PMid:16705963. http://dx.doi.org/10.1890/04-0576.

Littell, R. C., Milliken, G. A., Stroup, W. W., Wolfinger, R. D., \& Schabenberger, O. (2006). SAS for mixed models (2nd ed.). Cary: SAS Institute Inc.

Milliken, G. A., \& Johnson, D. E. (1992). Analysis of messy data (Vol. 1: Designed experiments). New York: Chapman \& Hall.

Missio, F., \& Jacobe, L. F. (2007). Variáveis Dummy: especificações de modelos com parâmetros variáveis. Ciência e Natura, UFSM, 29(1), 111-135.

Molenberghs, G., \& Verbeke, G. (2005). Models for discrete longitudinal data. New York: Springer.

Repola, J. (2006). Models for vertical wood density of scots pine, Norway spruce and birch stems, and their application to determine average wood density. Silva Fennica, 40(4), 673-685. http://dx.doi.org/10.14214/sf.322. 
Santos, P. E. T., Garcia, J. N., \& Geraldi, I. O. (2004). Posição da tora da árvore e sua relação com a qualidade de madeira serrada de Eucalyptus grandis. Scientia Forestalis, (66), 142-151.

SAS Institute Inc. (2012). Statistical Analysis System user's guide, Version 9.3. Cary: Statistical Analysis System Institute.

Searle, S. R., Casella, G., \& McCulloch, C. E. (1992). Variance components. New York: John Wiley \& Sons. http://dx.doi.org/10.1002/9780470316856.

Tomazello Filho, M. (1985). Variação radial da densidade básica e da estrutura anatômica da madeira do Eucalyptus saligna e E. grandis. Revista IPEF, (29), 37-45.

Verbeke, G., \& Molenberghs, G. (1997). Linear mixed models in practice: a SAS-oriented approach. New York: Springer. 306 p. http://dx.doi.org/10.1007/978-1-4612-2294-1.

Verbeke, G., \& Molenberghs, G. (2000). Linear mixed models for longitudinal data. New York: Springer.

Vonesh, E. F., \& Chinchilli, V. M. (1997). Linear and nonlinear models for the analysis of repeated measurements (560 p.). New York: Marcel Dekker.

Contribuição dos Autores: RAO: investigação, metodologia, escrita - primeira redação; GCB: conceituação, metodologia; DS: análise formal, escrita - revisão e edição; CRP: investigação, metodologia, escrita - revisão e edição . 